OAI-PMH: http://www.indteca.com/ojs/index.php/Revista Scientific/oai

Artículo Original / Original Article

\title{
Conocimientos y Aplicación de las TIC en la Educación Primaria Venezolana
}

Autores: Jerardin del Carmen Ríos Anciani Universidad Nacional Experimental "Rafael María Baralt", UNERMB jerardinrios@gmail.com Zulia, Venezuela https://orcid.org/0000-0003-4926-6435

Lucidio Segundo Hernández Ramírez Universidad Nacional Experimental "Rafael María Baralt", UNERMB hernandezlucidio@gmail.com Zulia, Venezuela https://orcid.org/0000-0002-0224-6560

\section{Resumen}

La investigación estuvo orientada a analizar el nivel de conocimiento en cuanto a la aplicación de las Tecnologías de Información y Comunicación por los docentes y directivos de educación primaria de la Parroquia José Cenobio Urribarrí, del Municipio Santa Rita del estado Zulia, Venezuela, como recurso didáctico. Epistemológicamente, se contextualiza dentro del enfoque cuantitativo, tipo de investigación analítica con un diseño no experimental, transaccional, y de campo. La población estuvo constituida por 65 personas (51 docentes y 14 directivos), utilizando criterio de censo poblacional. Se diseñaron 2 cuestionarios con escalamiento tipo Likert, con cuatro alternativas de respuesta, cuyo análisis fue realizado según la interpretación del promedio. Los resultados se ubican en una categoría moderada, con una baja dispersión, en las puntuaciones obtenidas de acuerdo con los indicadores establecidos, lo que indica que el nivel de conocimientos debe fortalecerse para que puedan utilizar y aplicar de manera efectivas las Tecnologías de la Información y la Comunicación (TIC) como recurso didáctico, ya que no ha sido asumida de manera efectiva.

Palabras clave: tecnología; conocimientos, información, comunicación, recurso didáctico.

Código de clasificación internacional: 5801.01 - Medios audiovisuales.

Cómo citar este artículo:

Ríos, J., \& Hernández, L. (2021). Conocimientos y Aplicación de las TIC en la Educación Primaria Venezolana. Revista Scientific, 6(20), 113-129, e-ISSN: 2542-2987. Recuperado de: https://doi.org/10.29394/Scientific. issn.2542-2987.2021.6.20.6.113-129

Fecha de Recepción: 02-12-2020
Fecha de Aceptación: 17-03-2021
Fecha de Publicación: 05-05-2021 
OAl-PMH: http://www.indteca.com/ojs/index.php/Revista Scientific/oai

Artículo Original / Original Article

\title{
Knowledge and Application of ICT in Venezuelan Primary Education
}

\begin{abstract}
The research was aimed at analyzing the level of knowledge regarding the application of Information and Communication Technologies by teachers and directors of primary education of the José Cenobio Urribarrí Parish, of the Santa Rita Municipality of the Zulia state, Venezuela, as a didactic resource . Epistemologically, it is contextualized within the quantitative approach, a type of analytical research with a non-experimental, transactional, and field design. The population consisted of 65 people (51 teachers and 14 directors), using the criteria of the population census. Two questionnaires were designed with Likert-type scaling, with four response alternatives, whose analysis was carried out according to the interpretation of the average. The results are located in a moderate category, with a low dispersion, in the scores obtained according to the established indicators, which indicates that the level of knowledge must be strengthened so that they can effectively use and apply Information Technology and Communication (ICT) as a didactic resource, since it has not been effectively assumed.
\end{abstract}

Keywords: technology; knowledge; information; communication; teaching resource.

International classification code: 5801.01 - Audio-visual methods.

\section{How to cite this article:}

Ríos, J., \& Hernández, L. (2021). Knowledge and Application of ICT in Venezuelan Primary Education. Revista Scientific, 6(20), 113-129, e-ISSN: 2542-2987. Recovered from: https://doi.org/10.29394/Scientific.issn.2542-2987.2021.6.20.6.113-129

Date Received: 02-12-2020
Date Acceptance: 17-03-2021
Date Publication: 05-05-2021 


\section{Introducción}

Las Tecnologías de Información y Comunicación (TIC), hoy en día están presentes en todos los espacios de la vida humana, en el ámbito familiar, laboral, social y principalmente el ámbito educativo, objeto de este artículo, haciendo énfasis en que las mismas han provocado cambios relevantes en la forma como el ser humano se comunica, se informa, se entretiene, de esta manera, la forma de enseñar y de aprender de las personas también ha cambiado adquiriendo nuevas perspectivas.

De acuerdo con González (2016): “[...] las TIC pueden ir más allá de ser herramientas tanto para la Información como para la Comunicación, constituyéndose en verdaderos elementos de apoyo en la formación educativa [...]" (pág. 495). Indudablemente estas herramientas tecnológicas poseen innumerables ventajas que pueden ser aprovechadas en beneficio de una mejor en la calidad educativa y en la formación de docentes que requieren de un manejo efectivo de las mismas para que garantice su integración adecuada dentro del proceso de enseñanza y aprendizaje.

En este sentido, en las instituciones educativas, en especial las de educación primaria, deben ser conocidas y adoptadas tanto por el personal directivo como por los docentes puesto que son herramientas valiosas que contribuyen a la formación integral de los individuos y constituyen un pilar fundamental dentro del Currículo Nacional Bolivariano. No obstante, pesar de que la inclusión de las tecnologías a la educación no es nada nuevo a esta fecha, aún existen profesionales docentes con debilidades en el manejo de la tecnología y más aún, poco se preocupan en formarse en el uso de estas.

En relación a lo anterior, la realidad en las escuelas, es que aun continua el modelo del profesor tradicional que utiliza como recursos la pizarra y la tiza, aun cuando en la institución posean portátiles Canaima, que pudieran ser utilizadas como un recurso por los niños, incluso los directivos se ven limitados al momento de utilizar el computador para realizar labores 
administrativas.

Lo anteriormente expuesto, puede ser producto posiblemente de una escasa formación en el área de la tecnología, se observa entonces resistencia al cambio, mientras los docentes niegan o retrasan el uso de las Tic aferrándose a las metodologías que están acostumbrados, y al parecer les resulta más fácil seguir utilizando los recursos que siempre han utilizado que abrirse a la posibilidad de conocer e integrar otros recursos como el uso de computadores, tabletas, e internet. Esto concuerda con lo expuesto por Viñals y Cuenca (2016): en referencia a que "existe un cierto temor ante el uso de las TIC e Internet y sus consecuencias" (pág. 109); dicho temor es una limitante para el uso efectivo de las herramientas informáticas.

Evidentemente, existe desconocimiento sobre los beneficios que estas herramientas aportan al proceso educativo como, la posibilidad de integrar texto, audio, e imágenes en un solo material, la inmediatez, el valor dinámico e interactivo, la innovación, además expande las posibilidades del docente en crear y diseñar sus propios recursos a través de los programas ofimáticos, y otros softwares destinados para ese fin, como por ejemplo: JClic Author y EdiLim. De igual manera en internet actualmente existen muchas páginas, blogs y wikis educativas con mucha información actualizada, atractiva y de interés tanto para educadores como los estudiantes.

De allí que, los beneficios para los estudiantes son innumerables, ya que el uso de estas tecnologías en el quehacer pedagógico permite la construcción de aprendizajes significativos dentro de un entorno dinámico, interactivo, creativo, armonioso, y que despierta el interés de los estudiantes, los motiva y anima a aprender. Con relación a lo anterior, Martínez (2018a), sostiene que:

Los contenidos y las estrategias administrados con el uso de las TIC, permiten al docente junto a sus pequeños educandos, socializar y adaptarse al tiempo y a las posibilidades de cada quien; donde el computador, la Internet, los simuladores de 
situaciones y sitios reales, y los juegos electrónicos educativos (de memoria, rompecabezas, completación, pareo, entre otros), son vías para el aprendizaje y el desarrollo del ser [...] (pág. 159).

Ahora bien, es importante destacar que los niños hoy día están en constante contacto con las tecnologías en su hogar, en su entorno, por ende, están familiarizados con el uso de las herramientas tecnológicas, porque han crecido rodeados de tecnología, parece insólito que los jóvenes estudiantes sean más hábiles que los docentes en el uso de la tecnología. No obstante, esa es una situación que puede ser aprovechada por los docentes, que aún no se han percatado de su importancia.

A esta realidad no escapan las instituciones educativas de la Parroquia José Cenobio Urribarrí, del Municipio Santa Rita del estado Zulia, Venezuela; donde se pudo percibir, mediante la observación directa en visita realizada a las instituciones objeto de estudio que los docentes poco incluyen el uso de las TIC en su quehacer pedagógico, manifestando que poseen poco conocimientos sobre estas herramientas, así como su integración en clase, aunado a esto, por otro lado los directivos aunque están conscientes de la relevancia de estas herramientas, no lo enfatizan con los docentes. En este sentido, Martínez (2018b), plantea que:

Los profesores no cuentan con una serie de competencias de cara a la utilización de las TIC, en cuanto a los contenidos y área de trabajo específicas se refiere; hecho que puede manifestarse negativamente no sólo en su labor formadora sino que además podría repercutir en sus planificaciones de aula [...] (pág. 160).

Como consecuencia de ello, se priva a los estudiantes de la posibilidad de construir saberes de manera integral, dinámica, creativa y los docentes se limitan a utilizar los mismos recursos, día tras día cayendo en la rutina y monotonía, lo que puede desmotivar a los estudiantes y hacer que sus clases 
en ocasiones sean aburridas.

La situación descrita, es motivada por el desinterés del docente en cuanto a la aplicación de las TIC, aunado a la escasa supervisión del directivo que no aplica los mecanismos necesarios para solventar esta situación, lo que contribuye a que prevalezca una educación poco innovadora, y un docente desactualizado, resistente al cambio.

Ahora bien, tomando en consideración la realidad descrita en los párrafos anteriores, se formuló como objetivo general de la investigación analizar el nivel de conocimientos del personal docente y directivo en las instituciones de educación primaria de la Parroquia José Cenobio Urribarrí, Municipio Santa Rita, estado Zulia, Venezuela, en cuanto a la aplicación de las TIC como recurso didáctico.

\subsection{Nivel de conocimientos sobre las TIC de docentes y directivos}

Hace referencia al conjunto de habilidades necesarias para el manejo efectivo de las herramientas tecnológicas, que le permitan a los usuarios de crear, editar, imprimir archivos, compartir textos, diseñar materiales, utilizando para ello aplicativos ofimáticos como los procesadores de texto, hojas de cálculo, bases de datos entre otras. El conocimiento sobre las TIC supone un elemento clave para el desarrollo personal y profesional" por lo tanto es de vital importancia considerar que dichos conocimientos son necesarios para impartir las clases en la sociedad del conocimiento que demanda una educación acorde con la realidad tecnológica del país.

En ese sentido, la acción del docente en especial quienes hacen actividad en las instituciones objeto de estudio, los conocimientos sobre el uso de las TIC debe estar dirigido a la integración teórica y práctica, puesto que de acuerdo a la información recabada, sus conocimientos están limitados a lo meramente básico y carecen de competencias para su utilización e inclusión efectiva, de allí que se extraen como indicadores, el nivel conceptual, nivel 
sensible (condicionado) y nivel integral (holístico).

A este respecto, Cabero (2010): desataca que "con las tic lo que debemos procurar es crear nuevas escenografías de aprendizaje, no reproducir las tradicionales [...]" (pág. 48); es decir, que no se trata de usar la tecnología para hacer las mismas cosas que hacíamos antes, con las tradicionales, el llamado es a innovar, a adquirir los conocimientos necesarios para integrar la tecnología y sus diferentes herramientas como parte de la gama de recursos que el docente tiene para trabajar.

De esta forma, es preciso destacar que los niños de hoy en día son activos, creativos, dados a la tecnología, requieren de un docente a la vanguardia de las nuevas tecnologías que propicie aprendizajes significativos, desde la innovación, razón por la cual, es necesario que la figura docente maneje un nivel de conocimientos adecuado para la aplicación efectiva de la tecnología, además de la buena disposición y sensibilización al respecto.

Ahora bien, en cuanto al nivel de conocimientos en el área conceptual se refiere al conocimiento y conceptualizaciones que se tienen en cuanto a una determinada temática, estos conocimientos pueden tener un nivel determinado de profundidad en el aprendiz, el conocimiento que tenga puede ser básico, moderado o avanzado, depende la profundidad y elaboración conceptual que tenga el individuo, puesto que en la práctica se ha formado y demostrado lo que conceptualmente (mental) ha desarrollado en cuando a las TIC, sus habilidades en el manejo de elementos conceptuales referidos a la herramienta.

En referencia a la sensibilidad, se hace mención la actuación del docente en el manejo y conocimiento de las TIC, como herramienta en su contexto educativo, quien asume diversas actitudes en cuanto a su manera ya de dar clases, a la que posiblemente está acostumbrado, se trata de ser sensible y consciente ante la posibilidad de abrirse a nuevos paradigmas y metodologías de trabajo. No obstante, el docente puede tener actitudes 
sensibles negativas para enfrentar de manera proactiva, los cambios en la metodología que exige la sociedad actual. De allí que, la disposición al cambio es una condición necesaria para poder avanzar hacia la sociedad el conocimiento y la información, representada por las herramientas tecnológicas, desde este punto de vista, la función docente debe ser innovadora, con estrategias acordes a la realidad que viven en el entorno, y adaptadas a las necesidades de los estudiantes, lo que se traduce en el mejoramiento de la calidad educativa.

Por otro lado, en nivel integral, partiendo desde el punto de vista de integralidad, la cual es definida como la capacidad de abarcar y unir lo social, psicológico, mental y físico en un solo aspecto. Este indicador, permite abarcar en el desarrollo de habilidades el manejo efectivo de las herramientas tecnológicas, así como también recursos multimedia, web, que coadyuven a la formación de sus estudiantes y fortalezcan su papel como docente.

En efecto, dentro de la integración de contenidos el docente como figura moderadora del aprendizaje, resaltando el papel activo del estudiante, debe utilizar las TIC como apoyo a la enseñanza con el fin de facilitar los procesos formativos de manera integral e interactiva. Esto concuerda con lo expresado por Castro, Guzmán y Casado (2007): quienes afirman que "las posibilidades que ofrecen las TIC, permiten al docente ser partícipe de la creación de entornos formativos en los cuales es eminente la interacción multidireccional entre los participantes, aumentando así la construcción de los aprendizajes" (págs. 220-221); de allí que el dialogo, y la participación activa de los actores es crucial, para la apropiación y renovación de saberes.

De manera tal, que los objetivos programáticos sean cónsonos con las estrategias a utilizar, aprovechando al máximo las ventajas de las nuevas tecnologías en el aula, para la obtención de resultados positivos. 


\subsection{Herramientas pedagógicas de las TIC como recurso didáctico}

Los recursos didácticos son medios, objetos y artefactos que facilitan el desarrollo de procesos de aprendizaje dinámicos dentro de las instituciones de educación, y las aulas de clase, es decir, que estos recursos se diferencian de otros tipos de materiales ya que se diseñan y utilizan para cumplir funciones vinculadas de manera específica con los procesos de enseñanza y aprendizaje. En este sentido, Calero, (2019), manifiesta que:

La introducción de las TIC en las aulas proporciona al profesorado innumerables herramientas que le ayudan a desempeñar su labor docente: recursos, medios, materiales didácticos, programas, etc., para extraer contenidos, diseñar las clases, facilitar el seguimiento del alumnado y su evaluación, etc. (pág. 32).

Bajo esta perspectiva, es preciso resaltar que, gracias a las TIC, los contenidos de las diferentes áreas académicas integradas, se presentan a los estudiantes en diferentes formatos, de manera atractiva, y en algunos momentos resulta ser un elemento clave dentro de la instrucción.

En este sentido, las herramientas de las Tic utilizadas por maestros y directivos son muy variadas, aunado a la necesidad que se tiene en la actualización de estas, puesto que la tecnología está en un constante cambio. No obstante, sobre la base de lo planteado y en función de lo observado en las escuelas de primarias en la Parroquia José Cenobio Urribarrí, Municipio Santa Rita, se concretan los siguientes indicadores descritos a continuación:

Un software se refiere a la parte intangible o lógica del computador, (los programas) que acompañan al hardware. El manejo de software por parte del usuario permite la creación, organización y diseño de todo tipo de actividades utilizando programas o aplicaciones que incluyen determinadas funcionalidades. 
Las herramientas ofimáticas constituyen un paquete de programas de trabajo como procesador de textos, hojas de cálculos, creación de diapositivas, dibujo y base de datos, además de otras aplicaciones que permiten crear, editar y almacenar datos. Aunado a lo anterior, se describen a continuación las herramientas TIC que son utilizadas como recursos didácticos en la educación:

La internet, está conformada por una red de computadoras las cuales se encuentran interconectadas entre sí a nivel mundial, a través de ellas se puede enviar y recibir información en cuestión de segundos a través de la www. Se utiliza para acceder a todos los servicios que proporciona internet: chat, correo electrónico, Navegación, web, entre otros.

El multimedia, constituye un conjunto de dispositivos compuestos por software y hardware, los cuales permiten la integración de manera simultánea de información en diferentes formatos de tipo: textual, gráfica, auditiva o de tipo icónica de interés para el usuario.

El ocio, se refiere a los programas, diseñados para el disfrute y la recreación bien sea offline u online, pueden ser programas de juego, video, y entretenimiento los cuales proporcionan al estudiante un entorno interactivo para aprender jugando.

La seguridad, componen los programas o recursos que tienen que ver con el margen de seguridad de los aparatos o dispositivos informáticos como Antivirus y anti-espías.

\section{Metodología}

La investigación de desarrolló dentro de la investigación cuantitativa, descriptiva, bajo el paradigma positivista, con un tipo de investigación analítica sobre la base de un diseño no experimental, transaccional, y de campo. La población estuvo constituida por 65 personas, 51 docentes y 14 directivos, utilizando como criterio el censo poblacional. Para la recolección de datos, se 
diseñaron dos cuestionarios, uno para directivos y otro dirigido a los docentes con escalamiento tipo Likert, con 5 alternativas de respuesta (5: Siempre; 4: Casi siempre; 3: A veces; 2: Nunca; 1: Casi Nunca), los cuales obtuvieron según el coeficiente alfa de Cronbach, un $96 \%$, lo que significa que ambos cuestionarios poseen un alto grado de confiabilidad.

\section{Análisis e interpretación de los resultados}

El presente análisis se hace en base a las respuestas dadas por docentes y directivos a quienes les fue aplicado. Para la interpretación de los resultados, se totalizaron los puntajes obtenidos de acuerdo con las alternativas de respuesta por cada ítem para luego agruparlos por dimensiones para su interpretación de acuerdo con el promedio; y la desviación estándar. Los resultados se detallan a continuación en la tabla 1.

Tabla 1. Nivel de conocimientos sobre las TIC.

\begin{tabular}{|l|c|c|c|c|}
\hline \multirow{2}{*}{\multicolumn{1}{c|}{ Indicadores }} & \multicolumn{2}{c|}{ Directiva } & \multicolumn{2}{c|}{ Docentes } \\
\cline { 2 - 5 } & Promedio & Desviación & Promedio & Desviación \\
\hline Conceptual & 3,40 & 0,26 & 3,08 & 0,26 \\
\hline Sensible (condicionado) & 3,38 & 0,27 & 3,04 & 0,18 \\
\hline Integral (Holístico) & 3,45 & 0,28 & 3,05 & 0,15 \\
\hline Promedio General & $\mathbf{3 , 4 1}$ & $\mathbf{0 , 2 7}$ & $\mathbf{3 , 0 5}$ & $\mathbf{0 , 2 0}$ \\
\hline
\end{tabular}

Fuente: Los Autores (2020).

En cuanto al Nivel de Conocimientos, se visualiza un promedio para la directiva de 3,41 , con una muy baja dispersión de 0,27 , ubicándose dentro de la categoría alta, afirmando de esta manera conceptualmente, tienen nociones sobre el uso de las TIC, y considerar se debe elevar el interés hacia su estudio, de manera tal que se transforme el aspecto condicionado de resistencia que hasta ahora presenta. Para los docentes, a través de sus respuestas se observa un promedio de 3,05 , con una muy baja dispersión de 0,20, ubicándose en una categoría moderada, ya que manifiestan poseer conocimientos de tipo conceptual con sensibilidad, es decir, que manejan los 
conocimientos básicos sobre las TIC dentro del aula, pero se ven limitados a la hora de obtener nuevos conocimientos de una manera más innovadora.

En referencia a lo propuesto, es preciso destacar que las habilidades deben ir de la mano con el conocimiento, puesto que ambos elementos son esenciales para el manejo de las TIC y que el aprendizaje sea significativo. Con relación a las herramientas utilizadas por docentes y directivos, los resultados se muestran a continuación en la tabla 2 .

Tabla 2. Herramientas de las TIC utilizadas como recurso didáctico.

\begin{tabular}{|l|c|c|c|c|}
\hline \multirow{2}{*}{\multicolumn{1}{c|}{ Indicadores }} & \multicolumn{2}{c|}{ Directiva } & \multicolumn{2}{c|}{ Docentes } \\
\cline { 2 - 5 } & Promedio & Desviación & Promedio & Desviación \\
\hline Software & 3,41 & 0,22 & 3,06 & 0,23 \\
\hline Ofimática & 3,41 & 0,28 & 3,06 & 0,23 \\
\hline Internet & 3,41 & 0,19 & 3,06 & 0,23 \\
\hline Multimedia & 3,41 & 0,19 & 3,06 & 0,23 \\
\hline Ocio & 3,41 & 0,19 & 3,06 & 0,23 \\
\hline Seguridad & 3,41 & 0,19 & 3,06 & 0,23 \\
\hline Promedio General & $\mathbf{3 , 4 1}$ & $\mathbf{0 , 2 2}$ & $\mathbf{3 , 0 6}$ & $\mathbf{0 , 2 3}$ \\
\hline
\end{tabular}

Fuente: Los Autores (2020).

Para la Dimensión, Herramientas de las TIC utilizadas como recurso didáctico se muestran los resultados obtenidos, de acuerdo con las respuestas de la directiva, donde se evidencia un promedio de 3,41, categoría alta con una muy baja dispersión de 0,22, lo que permite inferir que la directiva está consciente de la necesidad de aprender el manejo de las herramientas tecnológicas como es el Software Aplicativos, Animación, Internet, Multimedia, Ocio, Seguridad y la importancia de su implementación.

Sin embargo, en los docentes se obtuvo un promedio de 3,06 con una muy baja dispersión de 0,23 , lo cual se ubica en una categoría moderada, lo que indica que reconocen la importancia de las herramientas TIC en su quehacer laboral, y podrían estar dispuestos a aprender sobre ellas, más aún no la establecen dentro del aula. Sin duda alguna, es necesario asumir una postura flexible ante la importancia de las TIC dentro del quehacer didáctico. 


\section{Conclusiones}

Las tecnologías de Información y Comunicación llegaron para quedarse, poco a poco han transformado de manera drástica la forma de comunicarse, entre las personas y de igual manera la forma de enseñar y aprender también ha cambiado. Si bien los recursos tradicionales dieron buenos resultados a los docentes en su época, la realidad apunta a que ya no poseen la misma efectividad. Evidentemente, la incursión de las TIC en el ámbito educativo proporciona innumerables ventajas, tanto para el docente como los estudiantes, por hacer del proceso de aprendizaje una construcción dinámica e interactiva.

En consecuencia, no puede educarse en el siglo $X X$ como se educaba en el siglo XIX, por ende la escuela, como un espacio integral, activo debe proveer a los estudiantes una educación actualizada, acorde con los avances e innovaciones tecnológicas, en tal sentido es necesario una resignificación del papel del docente en cuanto a los roles que debe desempeñar, principalmente en nivel de conocimientos en el manejo de estas tecnologías debe considerarse desde el punto de vista conceptual, sensible e integral. A la vez, que debe manejar de manera efectiva las herramientas tic como un recurso didáctico dentro de su quehacer pedagógico.

No obstante, luego de haber realizado el análisis correspondiente al estudio se concluye que tanto el personal directivo como docente, en lo que respecta a las Tecnologías de la Información y la Comunicación (TIC), aún no está bien consolidado. En este sentido, las nuevas TIC son un pilar fundamental en la creación de la sociedad del conocimiento.

Ahora bien, los hallazgos obtenidos en la presente investigación aportan una información relevante al nivel de conocimientos del directivo que obtuvo un promedio de 3,41 , con una desviación estándar baja de 0,27 , ubicándose en una categoría alta. Por otro lado, en los docentes, se obtuvo un promedio de 3,05, con una muy baja dispersión de 0,20; ubicándose en una categoría 
moderada. Con relación a esto, queda claro que tanto el personal como directivo consideran manifiestan el dominio de conocimientos de tipo conceptual con sensibilidad, vale decir, que manejan los conocimientos básicos sobre las Tic dentro del aula, aunque se ven limitados al momento de aplicarlas, siendo necesario una mayor integración entre la teoría y la práctica.

En relación a la dimensión: para Herramientas de las TIC utilizadas como recurso didáctico, se obtuvo para la directiva, un promedio de 3,41 , ubicándose en una categoría alta, con una muy baja dispersión de 0,22, asimismo, para los docentes, se obtuvo un promedio de 3,06 , con una muy baja dispersión de 0,23 , ubicándose en una categoría moderada, lo que indica que ambos asumen la importancia de adquirir conocimientos sobre el manejo de herramientas tecnológicas, basadas en el uso de internet y computadores, aun cuando no las implementan en el aula.

Cabe destacar, que en la medida que el docente y directivo de las instituciones de educación primaria reciban y acepte el nuevo paradigma educativo basado en el uso de las TIC, que incluye la apropiación de nuevos conocimientos técnicos y aplicativos de las mismas, podrá entender que estas herramientas tecnológicas son un recurso, que complementa y facilita su labor.

\section{Referencias}

Cabero, J. (2010). Los retos de la integración de las TICs en los procesos educativos. Límites y posibilidades. Revista Perspectiva Educacional, 49(1), 32-61, e-ISSN: 0718-9729. Recuperado de: https://www.redalyc.org/articulo.oa?id=333327288002

Calero, C. (2019). La llegada de las nuevas tecnologías a la educación y sus implicaciones. IJNE: International Journal of New Education, 2(2), 21-39, e-ISSN: 2605-1931 Recuperado de:

http://doi.org/10.24310/IJNE2.2.2019.7449

Castro, S., Guzmán, B., \& Casado, D. (2007). Las Tic en los procesos de 
enseñanza y aprendizaje. Laurus, 13(23), 213-234, e-ISSN: 1315883X. Recuperado de:

https://www.redalyc.org/articulo.oa?id=76102311

González, M. (2016). Formación docente en competencias TIC para la mediación de aprendizajes en el proyecto Canaima Educativo. Telos, 18(3), 492-507, e-ISSN: 1317-0570. Recuperado de:

http://www.redalyc.org/articulo.oa?id=99346931009

Martínez, O. (2018a,b). Uso de las Tecnologías de la Información y la Comunicación en la Educación Básica. Revista Scientific, 3(10), 154174. Recuperado de: https://doi.org/10.29394/Scientific.issn.25422987.2018.3.10.8.154-174

Viñals, A., \& Cuenca, J. (2016). El rol del docente en la era digital. Revista Interuniversitaria de Formación del Profesorado, 30(2), 103-114, eISSN: 0213-8646. Recuperado de:

http://www.redalyc.org/articulo.oa?id=274473250 


\section{Jerardin del Carmen Ríos Anciani \\ e-mail: jerardinrios@gmail.com}

Nacida en Cabimas estado Zulia, Venezuela, el 4 de

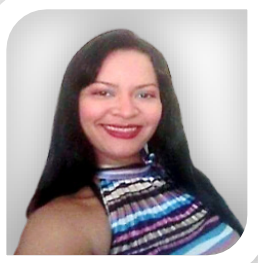
febrero del año 1985. Docente investigadora; obtuve mi título de Pregrado de Licenciada en Educación Integral en el año 2007 por la Universidad Nacional Experimental "Rafael María Baralt" (UNERMB); estudio de Postgrado con título de Magister Scientiarum en Administración de la Educación en el año 2015 por la Universidad Nacional Experimental "Rafael María Baralt"; tengo otros estudios de Técnico Superior Universitario en Informática, PNF Misión Sucre, en el 2019; cuento con experiencia laboral como Profesora Asesora Misión Sucre PNF Educación (2007-2010); Docente de Centros Bolivarianos de Informática y Telemática (CBIT), en la Escuela Básica Nacional "Santa Rita" (2008-2018); y Docente de Recursos para el Aprendizaje, desde el año 2008 hasta la actualidad, en la Unidad Educativa Nacional "Nuestra Señora del Rocío". 


\section{Hernández Ramírez Lucidio Segundo \\ e-mail: hernandezlucidio@gmail.com}

Nacido en Cabimas, Zulia, el 12 de abril del año 1973.

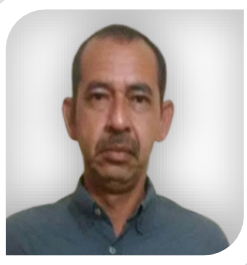

Docente Investigador; estudié en el Instituto Universitario de Tecnología Bomberil (IUTB), obteniendo el título de Licenciado en Ciencias del Fuego, Rescate y Seguridad; realicé mi Maestría en la Universidad Nacional Experimental "Rafael María Baralt", (UNERMB), obteniendo el título de Magister Scientiarum en Recursos Humanos; con experiencia laboral Docente como instructor en instituciones de educación y del Instituto Autónomo Cuerpo de Bomberos de Cabimas, Venezuela.

El contenido de este manuscrito se difunde bajo una Licencia de Creative Commons ReconocimientoNoComercial-Compartirlgual 4.0 Internacional 